\title{
Deflating the Deep Brain Stimulation Causes Personality Changes Bubble: the Authors Reply
}

\author{
Frederic Gilbert \& John Noel M. Viana \& C. Ineichen
}

\begin{abstract}
To conclude that there is enough or not enough evidence demonstrating that deep brain stimulation (DBS) causes unintended postoperative personality changes is an epistemic problem that should be answered on the basis of established, replicable, and valid data. If prospective DBS recipients delay or refuse to be implanted because they are afraid of suffering from personality changes following DBS, and their fears are based on unsubstantiated claims made in the neuroethics literature, then researchers making these claims bear great responsibility for prospective recipients' medical decisions and subsequent well-being. Our article "Deflating the 'DBS causes personality' bubble" reported an increase in theoretical neuroethics publications suggesting putative DBSinduced changes to personality, identity, agency, autonomy, authenticity and/or self (PIAAAS) and a critical lack of supporting primary empirical studies. This special issue of Neuroethics brings together responses to our initial publication, with our own counter-responses organized according to common themes. We provide a brief
\end{abstract}

F. Gilbert $(※)$ : J. M. Viana

Philosophy and Gender Studies Program, University of Tasmania, Hobart, Australia

e-mail: fredericgilbertt@gmail.com

J. M. Viana

Australian National Centre for the Public Awareness of Science, ANU College of Science, The Australian National University, Acton ACT 2601, Australia

C. Ineichen

Department of Psychiatry, Psychotherapy and Psychosomatics \& Department of Neurology, Psychiatric \& University Hospital Zurich, Zürich, Switzerland summary for each commentary and its main criticisms as well as a discussion of the way in which these responses can: 1) help clarify the meaning of PIAAAS, suggesting supplementary frameworks for understanding the impact of DBS on PIAAAS; 2) provide further empirical evidence of PIAAAS by presenting results from the researchers' own work; and/or 3) offer a critique of our research approach and/or findings. Unintended postoperative putative changes to PIAAAS remain a critical ethical concern. It is beyond dispute that we need to develop reliable empirical and conceptual instruments able to measure complex cognitive, affective, and behavioural changes in order to investigate whether they are attributable to DBS alone.

Keywords Agency · Authenticity · Autonomy - Deep brain stimulation $\cdot$ Identity $\cdot$ Personality $\cdot$ Self

Current philosophical and neuroethical discussions on the putative effects of deep brain stimulation (DBS) on personality, identity, agency, autonomy, authenticity and/or self(PIAAAS) turn on a crucial epistemological question: whether or not DBS causes unintended postoperative PIAAAS changes. Although the answer to this question does not always, it should always have a direct bearing on conclusions concerning what should (or should not) be ethically prescribed. Indeed, the very existence of a potential link between DBS and unintended postoperative PIAAAS changes suggests that certain moral obligations or prescriptions may be derived for these practices. For instance, medical and scientific authorities believe that 
frontal lobectomy contributes to unsafe irrevocable alteration of personality. Therefore, they have put in place bans and other measures to prevent this invasive brain intervention. But to be meaningful, our neuroethical prescriptions need to be based on epistemic certainties. In the case of frontal lobectomy, prevalence studies have indicated that the risk of unsafe irreversible personality changes, among other adverse effects, outweigh the benefits. Neuroethicists' discussions are sometimes referenced in public forums (e.g. the media) or honoured by individuals as having epistemic authority, generating conclusions that are rarely free of societal consequences. Prospective DBS recipients consulting and reading our discussions may be influenced by our conclusions when deciding whether to seek DBS surgery. If prospective DBS recipients delay or refuse to be implanted because they are afraid of suffering from personality changes following DBS, and their fear is generated from reading unsubstantiated conclusions in the neuroethics literature, then our conclusions bear great responsibility on the medical decisions and subsequent well-being of these patients. To conclude that there is enough or not enough evidence to show that DBS causes unwanted postoperative personality changes is an epistemic problem that should be answered on the basis of established, replicable, and valid data.

In our article "Deflating the 'DBS causes personality' bubble" [1], we found a critical lack of primary empirical studies supporting putative DBS-induced changes in PIAAAS, and we observed an increase in the number of theoretical neuroethics publications suggesting a link. Part of our approach involved critically assessing the evidence for these claims and conclusions. Given the low number of first-hand primary studies and large number of marginal and single case reports referenced in the theoretical neuroethics debates, we pointed out the existence of a DBS-PIAAAS bubble, which mayneed to be deflated due to limited empirical evidence.

This special issue of Neuroethics brings together responses to our initial publication [1], with our own counter-responses organized according to common themes. We provide a brief summary for each commentary and its main criticisms as well as a discussion of the way in which these responses can help further clarify, add evidence, and contribute to deliberations on how and to what extent DBS therapy affects the PIAAAS of recipients. We have grouped the responses according to whether they: 1) clarify the meaning of PIAAAS, suggesting supplementary frameworks for understanding the impact of DBS on PIAAAS; 2) provide empirical evidence of PIAAAS by presenting results from the researchers' own work; and/or 3) criticise our research approach and/or findings. A summary of each response, along with a classification according to its role orpurpose in the "DBS causes PIAAAS changes" debate is presented in Table 1.

\section{Clarifying the Meaning of PIAAAS}

A number of responses have provided additional frameworks in which the impact of DBS on PIAAAS can be analysed. For instance, Gaillard [2] presents two different levels of relevant analyses. Acknowledging that DBS is a technology that directly manipulates the brain, an organ essential for identity and personality, and simultaneously a medical intervention that modifies the living conditions of its recipients through symptom relief provides a basis for reconciling various narratives on how DBS causes PIAAAS changes and for conducting integrative research that fosters multiple perspectives via multidisciplinary approaches. In terms of facilitating integrative research to address the question of whether DBS causes unwanted PIAAAS changes, Kubu et al. [3] suggest the adoption of a pragmatic bioethical framework, which encourages open deliberation and empirical inquiry. Although the use of data from primary research involving DBS recipients is important, Pugh et al. [4] mention that there are cases when there is no first hand evidence to draw on for ethical analysis. In these instances, Pugh et al. [4] suggest the use of mechanism-based reasoning, which draws upon existing empirical work on the role of neural networks that are targeted by DBS, and the role that these networks play in psychopathology and human psychological traits.

Other responses have focused on specific aspects of PIAAAS and have suggested ways through which PIAAAS can be better operationalised. Erler [5] explains how the concepts of identity, authenticity, and autonomy are amenable to empirical inquiry. He highlights how philosophical conceptions of these terms can be used to design quantitative studies that capture changes to the DBS recipient's identity, authenticity, and autonomy. Zuk and Lazaro-Muñoz [6] echo a similar approach and discuss how traditional, experiential, and relational conceptions of autonomy can be used to develop an instrument that evaluates the direction, 
Table 1 Main arguments presented by responses to our article on "Deflating the Deep Brain Stimulation Causes Personality Changes Bubble" [1]

\begin{tabular}{lll}
\hline Author/s (Year) & Title & Purpose of response
\end{tabular}
Gaillard(2019) Neuroessentialism, our Technological
Future, and DBS Bubbles

Kubu, Ford, Wilt, Pragmatism and the Importance of

Merner,

Montpetite,

Zeigler, and

Racine (2019)

Erler (2019)

Bluhm and
$\begin{gathered}\text { It's Not Just Counting that Counts: a } \\ \text { Reply to Gilbert, Viaña, and } \\ \text { Ineichen }\end{gathered}$
Investigating Personality Changes Following DBS

Discussions of DBS in Neuroethics: Deflating Ethics?

\section{Clarifying PIAAAS: ways or narratives in which the effect of DBS on PIAAAS can be framed}

Clarifying PIAAAS: use of a pragmatic framework Can We Deflate the Bubble Without

\section{Clarifying PIAAAS: empirical investigation of autonomy, authenticity, and identity}

Criticism: ethical significance of these concepts despite lack of empirical grounding

Criticism: search strategy used; exclusion of case reports; limitation of quantitative instruments, randomised controlled trials, and incidence rates
The "DBS causes PIAAAS" bubble can be viewed through a brain narrative and/or a context narrative, with each narrative representing a different level in which DBS may exert its effects.

Contentions in neuroethics discussions could be a result of ethicists examining only one narrative and ignoring the other.

A pragmatic framework can be used to address the questions on whether DBS causes PIAAAS changes and to bridge conceptual and empirical neuroethics.

Results from their two previous studies show how DBS can improve patients' perceptions of control in their life and how PD is perceived to lead to more significantandnegative changes in personality than DBS.

Identity, authenticity, and autonomy are amenable to empirical inquiry through the help of philosophical conceptions of these constructs.

Some PIAAAS components may be irreducibly normative and cannot be fully operationalised or reduced; however, these do not make them less respectable concepts in neuroethics discussions.

The search terms severely restricted the initial set of results.

Case studies should not be dismissed, for they help inform theoretical papers that can illustrate important features and normative implications of DBS-related changes.

Randomised controlled trials are bad at identifying rare side effects and do not give information about factors that influence PIAAAS experiences.

PIAAAS instruments will not accurately reflect or capture patients' experiences.

Incidence rates tell very little about the nature of PIAAAS changes or the best way to help patients cope with them. 
Table 1 (continued)

\begin{tabular}{|c|c|}
\hline Author/s (Year) & Title \\
\hline $\begin{array}{l}\text { Pugh, Pycroft, } \\
\text { Maslen, Aziz, } \\
\text { and Savulescu } \\
\text { (2018) }\end{array}$ & $\begin{array}{l}\text { Evidence-Based Neuroethics, Deep } \\
\text { Brain Stimulation and Personality - } \\
\text { Deflating, but not Bursting, the } \\
\text { Bubble }\end{array}$ \\
\hline
\end{tabular}

Zuk and Lazaro-Muñoz (2019)

$\begin{array}{ll}\text { Mosley, } & \text { 'Woe Betides AnybodyWho Tries to } \\ \text { Robinson, } & \text { Turn me Down.' A Qualitative } \\ \text { Coyne, Silburn, } & \text { Analysis of Neuropsychiatric } \\ \text { Breakspear, and } & \begin{array}{l}\text { Symptoms Following Subthalamic } \\ \text { Carter(2019) }\end{array} \\ & \begin{array}{l}\text { Deep Brain Stimulation for } \\ \text { Parkinson's Disease }\end{array}\end{array}$

DBS and Autonomy: Clarifying the Role of Theoretical Neuroethics

Parkinson's Disease
Purpose of response

Clarifying PIAAAS: levels of evidence and mechanism-based reasoning to guide neuroethicists

Criticism: limitation of RCTs in detecting causality; mechanism-based reasoning suggests that DBS can affect authenticity in anorexia
Clarifying PIAAAS: scale for autonomy based on theoretical neuroethics

Empiricalevidence:impactofDBS for PD on autonomy, authenticity, and identity
Main arguments and/or findings

RCTs might not be able to tell us whether or not DBS directly causes personality changes; within-patient comparisons might provide evidence needed for the "DBS causes PIAAAS" question.

The most significant question is not about causality but rather the extent to which the effects of DBS on PIAAAS matter morally.

Neuroethical analyses on the effects of DBS should attend to relevant levels of evidence hierarchies, such as the one outlined by the Centre for Evidence-Based Medicine.

DBS for anorexia can have implications for authenticity, considering empirically-based mechanistic reasoning that accounts for features of $\mathrm{AN}$ and the brain regions being targeted for stimulation.

Developing an instrument for autonomy can be aided by understanding traditional, experiential, and relational conceptions of autonomy and their causal interdependence.

Presents 10 cases of people with PD who received DBS and developed behavioural and psychiatric symptoms, which were reduced after stimulation adjustment or lead repositioning. Three of these patients have no prior psychiatric history.

A qualitative interview of 10 patient-caregiver dyads demonstrates that actions or urges were a result of the brain being more active, and that DBS facilitated or allowed re-emergence of a younger, more energetic and extraverted, and real self that PD took away.

None of the participants considered that a change in identity had been precipitated by DBS, but some perceived that their autonomy had been overridden, especially for symptoms with negative consequences. 
Table 1 (continued)

\begin{tabular}{|c|c|c|}
\hline Author/s (Year) & Title & Purpose of response \\
\hline $\begin{array}{l}\text { Snoek, de Haan, } \\
\text { Schermer, and } \\
\text { Horstkötter } \\
(2019)\end{array}$ & $\begin{array}{l}\text { On the Significance of the Identity } \\
\text { Debate in DBS and the Need of an } \\
\text { Inclusive Research Agenda. A } \\
\text { Reply to Gilbert, Viana and } \\
\text { Ineichen }\end{array}$ & $\begin{array}{l}\text { Criticism: limitations of the search } \\
\text { strategy; inadequate evidence of } \\
\text { selective use of empirical findings } \\
\text { by ethicists; lumping of PIAAAS as } \\
\text { one; downplaying the contribution } \\
\text { of ethicists and qualitative research; } \\
\text { relevance of even a small risk of } \\
\text { PIAAAS changes }\end{array}$ \\
\hline
\end{tabular}

Main arguments and/or findings

There is little evidence provided on the claims that there is minimal empirical proof for PIAAAS changes due to DBS and that ethicists use empirical literature selectively, scantly, exaggeratedly, or out of context. For instance, de Haan's paper reports how OCD patients' self-experiences, relation to the world, and reflective attitudes change following DBS.

Not being able to establish a simple mono-causality should not be a ground to dismiss DBS as a factor that influences PIAAAS, and its corresponding ethical relevance.

The concepts of personality, identity, agency, autonomy, authenticity, and the self are lumped together by Gilbert et al., without properly defining each one and what qualifies as a change. It is also unfortunate that they concluded that PIAAAS should remain in the domain of philosophical instead of empirical research.

One could wonder if PIAAAS measures will be sufficiently developed, given that it is easy to mean different things using the same word.

Our bodies and our agency are not separate from each other, and thus, an altered body image is a threat to agency.

Gilbert et al. risk regressing the debate by proposing a hierarchy of sciences with certain quantitative methods on top and qualitative research and ethical analysis at the bottom. Doing this risks muting patient voices in disentangling the influence of DBS on PIAAAS.

Thomson, Changes in Personality Associated Segrave, and Carter (2019) with Deep Brain Stimulation: a QualitativeEvaluation ofClinician Perspectives
Empirical evidence: effect of DBS for Personality changes can be broadly movement and psychiatric disorders defined as changes in cognition, on personality

feeling, and behaviour.
Qualitative interview of 16 DBS clinicians for movement and psychiatric disorders showed that transient personality changes often occur within the first three months of DBS.

Clinicians working within psychiatric conditions did not describe 
Table 1 (continued)

\begin{tabular}{lll}
\hline Author/s(Year) & Title & Main arguments and/or findings \\
\hline & $\begin{array}{l}\text { changes in patient personality } \\
\text { following DBS. } \\
\text { Majority of clinicians believed that } \\
\text { changes in personality were } \\
\text { attributable to a variety of causes, } \\
\text { including the DBS, as well as } \\
\text { psychosocial, pathological and } \\
\text { pharmacological factors. }\end{array}$ \\
\hline
\end{tabular}

magnitude, and mechanism for changes to the three domains of autonomy in the DBS recipient. This instrument then provides an overall (autonomy) score that also incorporates a DBS recipient's particular view of autonomy. Indeed, more work needs to be done to disentangle what personality, identity, agency, authenticity, autonomy, and self really mean and to facilitate a collaborative discussion amongst academics from various disciplines, patients suffering from neurological or psychiatric disorders, and relatives and caregivers. Only by establishing a collaborative and multidisciplinary partnership can we develop instruments that comprehensively captures the incidence and extent of PIAAAS changes [17]. Results gained from such an integrated research approach would be useful in the informed consent process and in anticipating, mitigating, and managing any detrimental or unwanted effects associated with the influence of DBS on neural mechanisms.

\section{Providing Empirical Evidence about PIAAAS}

Several researchers present results from their own empirical studies to better delineate the extent of the effects ofDBS on PIAAAS. The qualitative interviews (involving 10 patient-caregiver dyads) by Mosley et al. [7] show how certain behavioural and psychiatric symptoms led to spousal caregiver burden and how these DBS-associated symptoms could affect attribution, responsibility, authenticity, and control. Interestingly, none of the people Mosley et al. [7] interviewed raised concerns about identity, which could signify that although DBS might lead to the expression of inauthentic behaviours, it does not lead to a shift in a person's underlying authentic selfhood. Kubu et al. [3] also share results from their work involving patients with $\mathrm{PD}$ (who received DBS) and their caregivers. Kubu and colleagues demonstrate how DBS can significantly improve patients' perceptions of control in vital aspects of their lives, particularly those reflecting their values. They also illustrate the need to view DBS-associated personality changes in light of personality changes due to $\mathrm{PD}$, considering that $\mathrm{PD}$ is associated with more negative personality changes while DBS is associated with more positive ones. Aligning with these findings, we believe it is probably a romantic view to see neurodegeneration in the substantia nigra as deteriorating only the motor system. It is no surprise that in recentyears, PD has more and more been characterized as a neuropsychiatric disorder rather than a neurological one [8], with potentially major implications for treatment and counselling. It is a similarly romantic view to believe that the effects of DBS can be confined to a very specific subset of neurons and that our anatomical and physiological knowledge of the underlying network-dynamics is at a sufficient level to have full control of what we modulate. Therefore, there is a fundamental need for studies to investigate the effects of DBS, PD, and other factors on PIAAAS. It is important to note that the findings of Kubu et al. [3] on how DBS affects control differ from those of Mosley et al. [7], who reported a perceived loss of control (or free will) in DBS recipients who viewed neuropsychiatric symptoms as inauthentic and attributable to DBS.

Thomson et al. [9] present the results of their qualitative interviews involving 16 clinicians working with DBS patients who suffered from a movementdisorder or psychiatric condition. Their results show that clinicians working on DBS for movement disorders reported personality changes months after surgery, with most being transient and easily addressable through clinical intervention. The transient nature of symptoms and their close temporal relationship with stimulation adjustment led to a number of clinicians directly attributing most 
personality changes to stimulation. However, other clinicians also acknowledged other factors such as medication and treatment outcome adjustments, disease progression, and psychosocial changes as contributors to observed personality changes. In contrast to clinicians working on DBS for movement disorders, clinicians working on psychiatric conditions did not describe patients' adverse events in terms of personality changes following DBS, because psychiatric DBS intends to change the patient's personality. These clinicians classified any changes in psychiatric symptoms as adverse events or restoration of the patient's pre-morbid personality. While acknowledging that DBS in the psychiatric context aims at intentionally changing the patient's personality, some of the main concerns that motivate the ethical and philosophical debates are about whether DBS may lead to unintended personality changes.

Overall, findings from the empirical studies of Thomson et al. [9], Mosley et al. [7], and Kubu et al. [3] demonstrate the potential diversity in PIAAAS changes experienced by people undergoing DBS therapy. They also highlight the need to acknowledge that unintended changes in aspects of PIAAAS, in particular personality, may be transient in nature and do not necessarily lead to drastic changes in a person's authentic selfhood and identity. They further argue that this illustrates that DBS, in certain cases, has the potential to restore, rather than disrupt or negatively alter, the PIAAAS of the DBS recipient $[10,11]$. This also aligns with recent quantitative and qualitative DBS studies suggesting that: personality changes were likely induced by postoperative tapering of dopaminergic treatment [12]; identity-related complaints were either transient or not associated with fundamental postoperative feelings of identity alteration [13]; and psychological variables did not undergo major changes [14]. Mosley et al. [7] and Thomson et al. [9] also state that indeed, DBS can cause personality changes, as evidenced by the close temporal relation between symptom onset or relief and stimulation adjustment; however, both groups do not disregard other contributing factors. The empirical contributions of Thomson et al. [9], Mosley et al. [7], and Kubu et al. [3] add new information to the debate on whether DBS may induce unintended PIAAAS changes. Although these studies were not conducted using a longitudinal, prospective, (sham)-controlled study design or even intra-individual DBS/sham comparisons-for this and other design considerations see e.g. [9]-they still provide important data on the nature and duration of DBS-associated PIAAAS changes. As will be outlined in the next section, the question of causality (i.e. does DBS cause unintended postoperative PIAAAS changes) needs to be approached in a rather narrow methodological sense. There are more methodological degrees of freedom when it comes to questions about the nature of PIAAAS changes that can "occur" after DBS implantation and stimulation, the way patients deal with them, among other important questions. In fact, these latter topics would tremendously benefit from a multidisciplinary approach.

\section{Addressing Criticisms of our Research Approach and Findings}

The primary aim of our paper [1] is to promote and encourage discussion of whether the portrayal of unintended DBS-induced PIAAAS changes in the academic literature is a bubble that needs to be deflated. Several authors have reacted to our findings and have challenged the claims we made. We have identified four major types of criticisms of our work and we will try to address them in this section.

First, a number of responses criticised our search strategy and the significance of our findings regarding the extent of empirical evidencejustifying philosophical claims that DBS causes unwanted PIAAAS changes. Bluhm and Cabrera [15] suggest that the search terms we used have severely restricted the initial set of results and have excluded a number of qualitative studies examining the experiences of DBS recipients. Snoek et al. [16] mention that we have limited evidence for our claim that there is minimal empirical proof for DBScaused unintended PIAAAS changes and that ethicists use empirical literature selectively, scantly, exaggeratedly, or out of context. They and Pugh et al. also point out that not being able to establish a simple monocausality should not be a ground to dismiss DBS as a factor that influences PIAAAS, and its corresponding ethical [16] and moral relevance [4].

We acknowledge the limitations of the search terms that we used; however, we would like to emphasise that we did not attempt to provide a systematic review on the subject matter and that we already acknowledged this limitation in our paper. We want to re-emphasise that the word "cause" implies scientific causation. To establish causality, one would have to test whether normalization of function can reverse pathological behaviour. Scientifically, this is far from a simple task as it requires complex study designs (as alluded to in our first 
publication) and sophisticated tools. Although we acknowledge the essential value of asking patients and family members whether they experienced PIAAASlike changes, as suggested by Snoek et al. [16], qualitative first-person perspectives are unfortunately not sufficient to scientifically establish causality. For causality testing, the undisputed concepts of necessity and sufficiency have been proposed (for a critique see [18, 19]). This would imply being able to test true ON-OFF conditions with as few as possible and as controllable as possible confounding factors (e.g. drugs, underlying disorder, sex, age). We refer to Montgomery for a thorough discussion of necessary and sufficient conditions [20] and only state that a necessary cause implies that the effect or the observation (e.g. change in PIAAAS) always co-exists with the cause (DBS) whilst a sufficient cause implies that each time the presumed cause (DBS) is present, the observation (PIAAAS) must be present. If DBS is neither a necessary nor a sufficient cause of PIAAAS, then it is epiphenomenal. The work of causality testing in DBS research in the context of PIAAAS has yet to be done and calls for meticulously planned study designs for understanding DBS's mechanisms of action. There is an important difference between saying that unanticipated changes in PIAAAS occur after DBS because of the technology and saying that these changes can occur in general. The question of causality not only makes a difference in discussions on a theoretical level but also in the context of counselling patients. Finally, we certainly do not mean to say that an unintended PIAAAS change is only ethically problematic if it results from DBS directly (mono-causality [16]); however, we believe that it is inherently problematic to specifically claim that "DBS causes PIAAAS" in the absence of robust empirical evidence that scientifically establishes causation, as highlighted above.

The second type of criticism is of our use of the acronym "PIAAAS" and our recommendation of developing instruments that would help measure the incidence and extent of PIAAAS changes. Snoek et al. [16] criticise our lumping together of personality, identity, agency, authenticity, autonomy, and self into a unitary concept (PIAAAS) without properly defining each component and what qualifies as a change. They also doubt the possibility of sufficiently developing PIAAAS measures given the multiple definitions and conceptions of personality, identity, agency, authenticity, autonomy, and self[16]. Erler [5] then points out that the limitations in fully operationalising or reducing
PIAAAS components does not make PIAAAS lessrespectable concepts used in discussions of neuroethics. Moreover, Bluhm and Cabrera [15] bring up how PIAAAS instruments will not accurately reflect or capture patients' experiences and how the incidence rates derived from these instruments would tell very little about the nature of PIAAAS changes or the best way to help patients cope with them.

We would like to clarify that we are not suggesting PIAAAS is a unitary concept in the neuroethics literature; rather, we use this acronym to refer to changes in personality, identity, agency, authenticity, autonomy, and/or self. Someone could have changes in personality without necessarily feeling or experiencing an altered selfhood, as suggested by the results of Mosley et al. [7]. We agree that it would indeed be challenging to develop certain PIAAAS measures, considering that even neuroethicists disagree among themselves on how to best define and encapsulate these terms. However, by no means should this deter efforts in developing instruments that can capture changes in PIAAAS. [17] To develop a framework for better understanding and measuring changes in PIAAAS during DBS (or any other medical intervention), we envision developing a strategy similar to the development of the Research Domain Criteria (RDoC) for understanding mental disorders. That strategy has aimed at de-emphasizing illness definitions as a starting point; instead, it seeks biological underpinnings and current understandings of behaviourbrain relationships and links them to clinical phenomenon $[21,22]$. Based on our (FG \& CI) experience of instrument development $[8,17,23,24]$, we are aware of the high demands that are needed for developing reliable and valid instruments that measure complex traits that indicate changes to PIAAAS. We are also aware that a quantitativePIAAAS instrument would not comprehensively reflect patients' experiences and the nature of any changes [17]. However, if we are to methodologically scrutinise the link between DBS and PIAAAS, we need instruments that can compare the extent and frequency of PIAAAS changes between patients who are undergoing DBS therapy and patients receiving standard or other clinical interventions.

The third type of criticism is associated with our point regarding how the responses "I feel like a robot" and "I feel like an electric doll" from Schupbach et al.'s [25] interviews do not necessarily indicate impaired autonomy, but rather, an altered body image. Snoek et al. [16] emphasise that our bodies and our agency 
are not separate from each other, and thus, an altered body image is a threat to agency. They state that the relationship between body and agency becomes apparent when the body stops functioning or regains lost capacities. To clarify, we are also not of the opinion that the body and agency are separate from each other. However, we would like to underscore that the statements from Schupbach's interviewees pertain more to body image - as a result of having a pulse generator and electrodes in them-than bodily function. Schupbach et al. (p. 1814; [25]) even propose that "the altered body image may result from difficulty in accepting psychologically the implanted material". Whether or not this altered body image has drastically affected the capacity of DBS recipients to act as agents is not discussed in Schupbach et al.'s [25] work. This highlights the need for us, as neuroethicists, to acknowledge the highly speculative nature of some of our claims, especially those based on short qualitative statements that have limited accompanying contextual information. As tempting as it is to interpret the statements regarding feeling like a robot or an electric doll as pertaining not just to an altered body image but also to the feeling of being controlled by the DBS apparatus, the only way to know if this really is the case is to review the original full-length interview transcript and/or interview again the DBS recipients who made such statements. Accordingly, one would have to determine if they also contextualised such mechanised feelings in terms of diminished capacity for agency.

The fourth and final set of criticisms pertains to our points regarding the methodological inadequacies of qualitative studies and case reports in determining whether DBS causes unanticipated PIAAAS changes and on how randomised controlled trials (RCTs) are well suited to address the causality question. Bluhm and Cabrera [15] point out that RCTs are bad at identifying rare side effects, in addition to not giving information about factors that influence PIAAAS experiences. Pugh et al. [4] also claim that RCTs might not be able to tell us whether DBS directly causes personality changes. Other researchers highlight the importance of not dismissing case studies, for they can help to inform theoretical papers of the normative implications of DBS-related changes [15]. Snoek et al. [16] warn that we risk regressing the debate by proposing a hierarchy of sciences with certain quantitative methods on top and qualitative research and ethical analysis at the bottom.
In reply, we first would like to underscore that we did not say that qualitative studies and ethical reflection are of minimal or subordinate value. Inquiries regarding the nature of PIAAAS changes, the extent to which they affect the lived experience of a patient receiving DBS and his/her caregivers, and the normative implications of such changes requires more than quantitative studies, and this is where qualitative research and case reports are fundamental for adequately comprehensive research. Furthermore, in no way do we want to propose a hierarchy of disciplines in understanding PIAAAS changes associated with DBS. As other responses to our article suggest $[5,6]$, philosophy is an essential aid in the clarification of terms and in the development of clinical instruments. However, we would like to re-emphasize: contrary to some of the claims made in response to our article, we are of the firm opinion that there is a need for scientific study designs to answer the "causality" question because apart from theoretical discussions about the logic(s) of causality, this question needs to be addressed by empirical experiments that use appropriate study designs capable of disentangling DBS effects from potential confounders. In recruiting participants, designing the study, and statistically analysing the results, RCTs can control and account for other factors leading to PIAAAS changes, including but not limited to disease type, duration, and severity; stimulation parameters; and medication regime. Furthermore, the capacity of an RCT to identify rare effects ultimately depends on the sample - and effect size of the underlying construct. If certain PIAAAS changes are indeed rare, then this could actually represent a bubble in terms of how their prevalence is portrayed.

We would also like to point out that although the within-patient study design proposed by Pugh et al. [4] has its appeals, it may face real-world issues, such as the fact that we cannot assume that PIAAAS-changes can be modulated instantly and therefore could not easily be tested in ON vs OFF conditions. DBS may be associated with long-term synaptic changes [26] that cannot be undone by simply turning off the stimulation. Changes in drugs that often have been used over decades further complicates the issue. Hence, while we agree that approaching the question of causality is difficult, there are study designs, in this regard, that are clearly better and yield more robust information. Pre-clinical causality testing using animal models of DBS might provide potential support as well. If it were true that DBS causes unwanted PIAAAS, this information would be of great 
clinical significance, given the advent of alternative modulation possibilities through current steering, stimulation adjustment, or in some cases, lead repositioning [7] and other neuromodulation techniques.

Overall, we acknowledge the importance of multiple perspectives and open deliberation [3] in discussing whether DBS is associated with PIAAAS, and we hope that this section serves as a starting point for further conversations and investigations on this topic. Hopefully, future empirical work on this topic will also provide theoretical neuroethicists with additional material to reflect on, facilitating more comprehensive and evidence-based discussions regarding the extent of the effects of DBS on unintended PIAAAS and on their relevance to DBS recipients, their caregivers, and the attending medical team.

\section{Conclusion}

This special issue provides a unique opportunity for interdisciplinary researchers examining unintended DBS-associated PIAAAS changes to reflect on the issue in light of the evidence that we presented in our paper, "Deflating the 'DBS causes personality changes' bubble" [1]. We greatly appreciate the various commentaries provided which assess the validity and significance of our initial arguments [1]. We hope this special issue will be a starting point for fostering a fruitful collaboration among neuroethicists from various disciplines, philosophers, medical personnel treating patients with neurological and/or psychiatric conditions, and patients and their caregivers [27-45].

We neuroethicists should be careful in endorsing any conclusion, positive or negative, about whether DBS causes unintended PIAAAS changes. A precautionary conclusion might be to subscribe to an agnostic view. Any conclusion drawn should be based on replicable and valid data. Furthermore, when advocating for a conclusion, we should use language that is patientcentred, especially since it may then be more useful for helping to guide patients to make decisions aligning with their personal values and preferences. The difficulty in concluding whether DBS causes unintended PIAAAS changes is great because of both an underlying measurement problem (i.e. the frequency of complex side effects is difficult to estimate) and an evaluation problem (i.e. the retrospective assessment can significantly differ between patients, their relatives, and physicians) [46]. Unintended postoperative putative changes to PIAAAS remain a critical ethical concern. It is beyond dispute that we need to develop reliable and valid instruments able to measure complex cognitive, affective, and behavioural changes postoperatively in order to investigate whether changes in PIAAAS are attributable to DBS alone. We too need to uphold what all professionals in the field ultimately strive for: adherence to the highest scientific standards. Equally critical is a need for more empirical and wellgrounded conceptual neuroethics discussions on the topic of DBS and PIAAAS. At stake are profound implications for patients' decisions and lives. It is not enough to say that DBS may result in changes in PIAAAS. Instead we must strive to indicate the extent, in terms of magnitude and persistence, of these changes and the availability of strategies to mitigate them. Achieving this will require no less than the tools, knowledge, and perspectives of multiple disciplines. Neuroethics, both empirical and conceptual, is a field that is inherently multidisciplinary. Only by fostering an open dialogue can we ensure that relevant discussions in the ethics of neuroscience are properly grounded and do not become an impenetrable bubble detached from empirical evidence and from the actual lived experience of the very people whose lives are accordingly so significantly affected.

\section{References}

1. Gilbert, F., J.N.M. Viaña, and C. Ineichen. 2018. Deflating the "DBS causes personality changes" bubble. Neuroethics. https://doi.org/10.1007/s12152-018-9373-8.

2. Gaillard, M. 2019. Neuroessentialism, our technological future, and DBS bubbles. Neuroethics. https://doi. org/10.1007/s12152-019-09407-6.

3. Kubu, C.S., P.J. Ford, J.A. Wilt, A.R. Merner, M. Montpetite, J. Zeigler, and E. Racine. 2019. Pragmatism and the Importance of Interdisciplinary Teams in Investigating Personality Changes Following DBS. Neuroethics. https://doi.org/10.1007/s12152-019-09418-3.

4. Pugh, J., L. Pycroft, H. Maslen, T. Aziz, and J. Savulescu. 2018. Evidence-based neuroethics, deep brain stimulation and personality - deflating, but not bursting, the bubble. Neuroethics. https://doi.org/10.1007/s12152-018-9392-5.

5. Erler, A. 2019. Discussions of DBS in neuroethics: Can we deflate the bubble without deflating ethics? Neuroethics. https://doi.org/10.1007/s12152-019-09412-9.

6. Zuk, P., Lázaro-Muñoz, G.: DBS and autonomy: Clarifying the role of theoretical neuroethics. Neuroethics (2019). doi: https://doi.org/10.1007/s12152-019-09417-4 
7. Mosley, P.E., Robinson, K., Coyne, T., Silburn, P., Breakspear, M., Carter, A.: 'Woe betides anybody who tries to turn me down.' A Qualitative Analysis of Neuropsychiatric Symptoms Following Subthalamic Deep Brain Stimulation for Parkinson's Disease. Neuroethics (2019). https://doi.org/10.1007/s12152-019-09410-x

8. Ineichen, C., H. Baumann-Vogel, and M. Christen. 2016. Deep brain stimulation: in search of reliable instruments for assessing complex personality-related changes. Brain sciences 6 (3): 40.

9. Thomson, C.J., R.A. Segrave, and A. Carter. 2019. Changes in personality associated with deep brain stimulation: A qualitative evaluation of clinician perspectives. Neuroethics. https://doi.org/10.1007/s12152-019-09419-2.

10. Ardouin, C., V. Voon, Y. Worbe, N. Abouazar, V. Czernecki, H. Hosseini, A. Pelissolo, E. Moro, E. Lhommee, A.E. Lang, Y. Agid, A.L. Benabid, P. Pollak, L. Mallet, and P. Krack. 2006. Pathological gambling in Parkinson's disease improves on chronic subthalamic nucleus stimulation. Mov Disord21 (11): 1941-1946. https://doi. org/10.1002/mds.21098.

11. Bewernick, B.H., R. Hurlemann, A. Matusch, S. Kayser, C. Grubert, B. Hadrysiewicz, N. Axmacher, M. Lemke, D. Cooper-Mahkorn, M.X. Cohen, H. Brockmann, D. Lenartz, V. Sturm, and T.E. Schlaepfer. 2010. Nucleus accumbens deep brain stimulation decreases ratings of depression and anxiety in treatment-resistant depression. Biol Psychiatry 67 (2): 110-116. https://doi.org/10.1016/j. biopsych.2009.09.013.

12. Lhommée, Eugénie, et al. 2017. Personality, dopamine, and Parkinson's disease: Insights from subthalamic stimulation. Movement Disorders 32 (8): 1191-1200.

13. Baertschi, M., N. Favez, M. Radomska, et al. 2019. An empirical study on the application of the burden of normality to patients undergoing deep brain stimulation for parkinson's disease. J Psychosoc Rehabil Ment Health 6: 175-186. https://doi.org/10.1007/s40737-019-00149-5.

14. Baertschi, M., et al. 2020. Illness Representations and Coping Strategies in Patients Treated with Deep Brain Stimulation for Parkinson's Disease, Journal of Clinical Medicine, 9 (4). https://doi.org/10.3390/jcm9041186.

15. Bluhm, R., and L.Y. Cabrera. 2018. It's not just counting that counts: A reply to Gilbert, Viaña, and Ineichen. Neuroethics. https://doi.org/10.1007/s12152-018-9391-6.

16. Snoek, A., de Haan, S., Schermer, M., Horstkötter, D. 2019.: On the Significance of the Identity Debate in DBS and the Need of an Inclusive Research Agenda. A Reply to Gilbert, Viana and Ineichen. Neuroethics. https://doi.org/10.1007 /s12152-019-09411-w.

17. Gilbert, F., Brown, Dasgupta et al. 2019. An instrument to capture the phenomenology of implantable brain device use Neuroethics. https://doi.org/10.1007/s12152-019-09422-7.

18. Yoshihara, M., \& Yoshihara, M. 2018. 'Necessary and sufficient' in biology is not necessarily necessary-confusions and erroneous conclusions resulting from misapplied logic in the field of biology, especially neuroscience. $J$ Neurogenet. 32(2), 53-64.

19. Gomez-Marin, A. 2017. Causal circuit explanations of behavior: Are necessity and sufficiency necessary and sufficient? In Decoding Neural Circuit Structure and Function (pp. 283-306). Cham: Springer.
20. Montgomery E.B. 2012. The epistemology of Deep Brain Stimulation and neuronal pathophysiology. Front Integr Neurosc. 6.

21. Insel, Thomas, et al. "Research domain criteria (RDoC): toward a new classification framework for research on mental disorders." (2010): 748-751.

22. Cuthbert, Bruce N., and Thomas R. Insel. 2013. Toward the future of psychiatric diagnosis: the seven pillars of RDoC. BMC medicine 11 (1): 126.

23. Ineichen, C., M. Christen, and C. Tanner. 2017. Measuring value sensitivity in medicine. BMC medical ethics 18 (1): 5.

24. Bouthour, W., P. Mégevand, J. Donoghue, C. Lüscher, N. Birbaumer, and P. Krack. 2019. Biomarkers for closed-loop deep brain stimulation in Parkinson disease and beyond. Nature Reviews Neurology 1.

25. Schupbach, M., M. Gargiulo, M.L. Welter, L. Mallet, C. Behar, J.L. Houeto, D. Maltete, V. Mesnage, and Y. Agid. 2006. Neurosurgery in Parkinson disease: a distressed mind in a repaired body? Neurology 66 (12): 1811-1816. https://doi.org/10.1212/01.wnl.0000234880.51322.16.

26. Herrington, T.M., J.J. Cheng, and E.N. Eskandar. 2016. Mechanisms of deep brain stimulation. JNeurophysiol 115 (1): 19-38. https://doi.org/10.1152/jn.00281.2015.

27. Pugh, J. 2020. Clarifying the normative significance of 'personality changes' following deep brain stimulation. Sci Eng Ethics. https://doi.org/10.1007/s11948-020-00207-3.

28. Gilbert, F., and M. Lancelot. 2020. Incoming ethical issues for Deep Brian Stimulation: When long term treatment lead to a "new form of disease". Journal of Medical Ethics. https://doi.org/10.1136/medethics-2019-106052.

29. Stevens, I., and F. Gilbert. 2020. N-of-1 trial for closed-loop deep brain stimulation devices. Ethics \& Human ResearchThe Hasting Center. https://doi.org/10.1002/eahr.500045.

30. Horstkötter, D., and G. de Wert. 2020. Ethical considerations. In Fundamentals and clinics of deep brain stimulation, ed. Y. Temel, A. Leentjens, R. de Bie, S. Chabardes, and A. Fasano. Cham: Springer.

31. Stevens, I., and F. Gilbert. 2020. Experimental usage of AI brain-computer interfaces: computerized errors, side-effects, and alteration of personality. In Ethics of medical innovation, experimentation, and enhancement in military and humanitarian contexts, ed. D. Messelken and D. Winkler. Cham: Military and Humanitarian Health Ethics. Springer.

32. Goering S, Sullivan LS, Introduction to the special section: feminist approaches to neurotechnologies. IJFAB, 13 Issue 1, Spring 2020, pp. 89-97. https://doi.org/10.3138 /ijfab.13.1.05

33. De Marco, G. 2019. Brain interventions, moral responsibility, and control over one's mental life. Neuroethics 12: 221229. https://doi.org/10.1007/s12152-019-09414-7.

34. Gilbert, F., and J.M.N. Viaña. 2018. A Personal narrative on living and dealing with psychiatric symptoms after DBS surgery. Narrative Inquiry in Bioethics. 8 (1): 67-78.

35. Bluhm, R., L. Cabrera, and R. McKenzie. 2019. What we (should) talk about when we talk about deep brain stimulation and personal identity. Neuroethics. https://doi. org/10.1007/s12152-019-09396-6.

36. Dings, R Not being oneself? Self-ambiguity in the context of mentaldisorder, Printed by ProefschriftMaken, 2019, ISBN: 978-94-6380-573-5. 
37. Gilbert, F., E. Goddard, J.M.N. Viaña, A. Carter, and M. Horne. 2017. "I miss being me": Phenomenological effects of deep brain stimulation. American Journal of Bioethics Neuroscience 8 (2): 96-109. https://doi.org/10.1080 $/ 21507740.2017 .132031938$.

38. Ramirez-Zamora, A., et al. 2020. Proceedings of the seventh annual deep brain stimulation think tank: advances in neurophysiology, adaptive dbs, virtual reality, neuroethics and technology. Front Hum Neurosci. 14: 54. https://doi. org/10.3389/fnhum.2020.00054.

39. Miletic, T., Gilbert F. (2020) Does AI brain implant compromise agency? Examining Potential harms of braincomputer interfaces on self-determination. In Artificial intelligenceandinformation:a multidisciplinaryperspective.Eds S.S. Gouveia \& J.F. Teixeira. Vernon Press.

40. Viaña, J.M.N., and F. Gilbert. 2018. Deep brain stimulation for people with alzheimer's disease: anticipating potential effects on the tripartie self. Dementia: International journal of social research and practice. 18 (7-8): 2836-2855. https://doi.org/10.1177/1471301218761147.

41. Walker, JW, Mackenzie, C. Neurotechnologies, relational autonomy, and authenticity. IJFAB, 13 Issue 1, Spring 2020, pp. 98-119 10.3138/ijfab.13.1.06

42. Gilbert, F. 2018. Deep brain stimulation: inducing self-estrangement. Neuroethics 11: 157-165. https://doi. org/10.1007/s12152-017-9334-7.
43. Goddard, E. Relational Agency and Neurotechnology: Developing and Deploying Competency through Intricate Partnerships. IJFAB, 13 Issue 1, Spring 2020, pp. (2020) https://doi.org/10.3138/ijfab.13.1.11

44. Gilbert, F. 2012. The burden of normality: From 'chronically ill' to 'symptom free'. New ethical challenges for deep brain stimulation postoperative treatment. J Med Ethics. 38: 408412. https://doi.org/10.1136/medethics-2011-100044.

45. Marc Baertschi, João Flores Alves Dos Santos, Pierre Burkhard, Kerstin Weber, Alessandra Canuto, Nicolas Favez. 2019. The burden of normality as a model of psychosocial adjustment after deep brain stimulation for Parkinson's disease: A systematic investigation. Neuropsychology. 33 (2):178-194

46. Müller, S., and M. Christen. 2011. Deep brain stimulation in Parkinsonian patients - Ethical evaluation of cognitive, affective, and behavioral sequelae. AJOB Neuroscience.2 (1): 3-13.

Publisher's Note Springer Nature remains neutral with regard to jurisdictional claims in published maps and institutional affiliations. 